Part 1. The Star Clusters of Local Group Galaxies Section A. Invited Reviews and Contributed Talks 


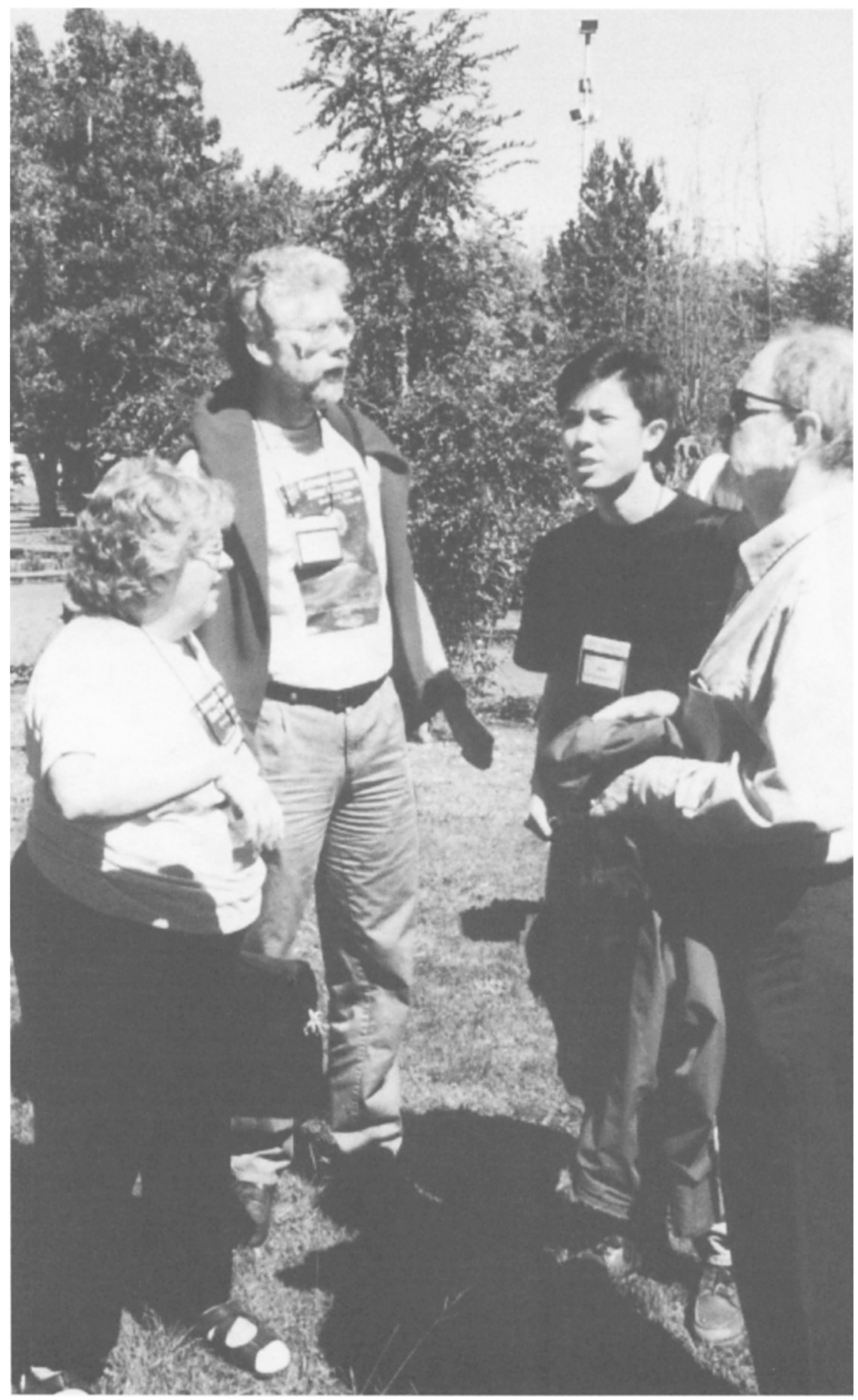

Gretchen and Bill Harris, Eric Peng and Jim Hesser preparing for the conference photo in the plaza. 
Doug Geisler, Eva K. Grebel, and Dante Minniti, eds.

\title{
Metal-Poor Globular Clusters of the Milky Way and Environs
}

\author{
James E. Hesser and Jon P. Fulbright
}

Dominion Astrophysical Observatory, Herzberg Institute of Astrophysics, National Research Council, 5071 W. Saanich Road, Victoria, BC V9E 2E7, Canada

"He aquí los astros pálidos todos llenos de enigma" Pablo Neruda, El hondero entusiasta

\begin{abstract}
We review properties of the most metal-deficient globular clusters and halo stars in the local universe as of March 2001, with goals of examining present evidence for the duration of the initial burst of massive cluster formation in the Milky Way, determining when that burst occurred, and elucidating what appears to be limiting our present understanding. Such issues bear upon many topics to arise later in the Symposium.
\end{abstract}

\section{Introduction}

The most metal-deficient stars of the local universe provide an unparalleled opportunity to study material representative of a much earlier epoch, perhaps predating the formation of the prominent components of the highest redshift galaxies being discovered today. While the massive globular star clusters (GCs) offer distinct, well-known advantages in such research, individual stellar chemistry is a powerful tool in the quest to understand early Galactic history. Unusually comprehensive recent review volumes are those of Martínez Roger, Pérez Fournón \& Sánchez (1999), Carney (2001) and Harris (2001).

As is well known, the metallicity distribution of the 137 Milky Way GCs with $[\mathrm{Fe} / \mathrm{H}]$ determinations is well described by two Gaussian curves centered on $[\mathrm{Fe} / \mathrm{H}]=-1.6$ and -0.6 (Harris 2001) on the popular Zinn \& West (1984) metallicity scale. Of particular interest in this context is that the most metaldeficient clusters have $[\mathrm{Fe} / \mathrm{H}] \sim-2.3$, whereas the most metal-deficient halo stars extend to metallicity values more than an order of magnitude lower (cf., Carney et al. 1996). While not entirely a proper comparison, the most metal-poor GCs and halo stars exhibit compositions well below those of damped Lyman- $\alpha$ forest systems at $z>3$ (cf., Prochaska \& Wolfe 2000), yet ironically many of the extremely metal-deficient Galactic objects likely to have formed at high redshift can be seen with binoculars.

Other properties of the GC system offer important clues to its origin. At Galactocentric radii $\left(R_{\mathrm{gc}}\right)$ beyond $10 \mathrm{kpc}$, the average $M_{v}$ of those clusters with 
blue HB morphology is -7.3 , while clusters exhibiting red horizontal-branch (HB) morphology have a corresponding value of -4.8 (van den Bergh 1996). Half-light radii for Galactic GCs show a dependence on Galactocentric radii, with the farthest ones generally being larger in diameter. While exhibiting similar trends, the clusters of the Fornax dSph or the LMC have a different distribution (van den Bergh 2000a). Van den Bergh (2000a,b) interprets the present data as evidence that the Galaxy, Fornax and the LMC were distinct systems when they started to form their GCs: it appears that somehow the GCs "knew" they were going to end up in their respective parent galaxy.

By correlating the spatial distributions and kinematics of Galactic halo tracers, Hartwick (2000) argues that the spatial distribution of Milky Way companion galaxies define a real triaxial structure reflecting the initial conditions of the Galaxy's formation epoch. The outer halo GCs form a nearly oblate, flattened system whose minor axis is highly inclined to the present rotation axis, and thus do not seem part of the same triaxial system.

\section{Length of Initial Burst}

How long did the initial burst of cluster formation last for the Milky Way? The answer, from relative ages of the most metal-deficient halo stars, can help distinguish among conceivable formation scenarios (e.g., fast collapse, leisurely buildup through mergers, etc.). While relative measurements are usually more reliable to make and more straightforward to interpret, controversy about relative cluster ages persists.

Morphological properties of HBs have been extensively explored for their potential as a powerful tool for sensitive age discrimination among clusters. From plots of metallicity against the ratio of the difference in the number of blue and red $\mathrm{HB}$ stars to the total $\mathrm{HB}$ population, compared with isochrones from synthetic HB models for GCs, Y.-W. Lee, Demarque, \& Zinn (1994, et seq.; hereafter LDZ) found evidence for an age range of about 4 Gyrs, or some $25-35 \%$ of the age of a 12-15 Gyr old Galaxy. The age range so inferred is present at all metallicities, and an age- $R_{\mathrm{gc}}$ relation was suggested. The concepts which emerged from this important work have exerted wide influence in thinking about how long it took to form or assemble the Galactic halo. Many accept that evidence from GCs strongly favors a relatively drawn out formation, which fits nicely with growing support for the importance of hierarchical mergers during the formation of large galaxies. However, this compelling interpretation of $\mathrm{HB}$ morphologies has been the subject of debate over the past seven or so years. In part this arises because stellar evolutionary models indicate that $\mathrm{HB}$ morphology is sensitive to differences in parameters that are well below present observational uncertainties. Specific counterexamples to the LDZ analysis noted by, e.g., Stetson, VandenBerg, \& Bolte (1997), are disconcerting. What seems to be the current situation?

Harris et al. (1997) compared comparably deep (V,V-I) color-magnitude diagrams (CMDs) for the massive GCs M92 and NGC 2419, which are among the most metal-deficient GCs in the Galaxy. They were unable to detect any age difference in excess of 1 Gyr. Importantly, M92 resides in the inner part of the Galactic halo, while NGC 2419's $\mathrm{R}_{\mathrm{gc}} \sim 90 \mathrm{kpc}$ places it in the outermost 
halo (roughly twice as far as the LMC). Moreover, its large core and tidal radii are a strong indication that it has existed in the outer halo throughout its life, for otherwise dynamical forces would have modified its appearance. (Note that Shetrone, Côté, \& Sargent 2001 have recently determined, for the first time, a metallicity for NGC 2419 from high-dispersion spectra of giants which confirms the validity of pairing it with M92). This single comparison among archetypical metal-deficient clusters from different regions of the halo already provides stringent constraints on the duration of the formation of the most massive clusters early in Galactic (pre)history. Grundahl's (2001) comparisons of deep CMDs for M92, NGC 5053 and NGC 5466 in the Strömgren photometric system similarly found no evidence of age differences in excess of $\sim 1$ Gyr.

An extensive study by Rosenberg et al. (1999) applied both techniques of relative age determinations, the color-difference (between the lower giant branch and the turnoff) and luminosity-difference (between the turnoff and the HB), to a homogeneous data set for $35 \mathrm{GCs}$. They found all the clusters with $[\mathrm{Fe} / \mathrm{H}]<-1.2$ dex to be old and coeval. Some clusters in the range $-1.2 \leq[\mathrm{Fe} / \mathrm{H}] \leq-0.9 \mathrm{dex}$ appear to be up to $25 \%$ younger than the otherwise co-eval oldest clusters, while the most metal-rich clusters studied appear to be co-eval. They infer that the GC formation process started at the same time throughout the halo to a distance of $\sim 20 \mathrm{kpc}$ from the Galactic center, with the small population of significantly younger halo clusters found at $R_{g c}>8 \mathrm{kpc}$ likely the result of later mergers. They further note that their evidence for the co-eval metal-rich clusters being $\sim 17 \%$ younger than other GCs (that is, for a mild age-metallicity relation) is particularly model dependent. J.-W. Lee et al. (2001) have extended the Rosenberg et al. analysis with three extremely metal-poor clusters located in the innermost Galactic halo, further strengthening the evidence against an age range or of a trend in ages with $R_{g c}$ among the most metal-deficient GCs in the Galaxy.

On the question of the existence of an age-metallicity relation, VandenBerg (2000) analyzed in a systematic manner high-quality CMD data from the literature for $26 \mathrm{GCs}$. He infers that unless $[\mathrm{O} / \mathrm{Fe}]$ or $[\alpha / \mathrm{Fe}]$ are up significantly at the lowest metallicities, then a mild age-metallicity relation results. At any given metallicity, however, he found the data do not permit an age range $\geq 10-15 \%$.

The famous "second-parameter" GCs at intermediate metallicities and large $\mathrm{R}_{\mathrm{gc}}$ figure prominently in efforts to understand Galactic halo formation. Their great distances, and low masses (hence sparse CMDs) have challenged observations from the ground. Stetson et al. (1999) reported relative age comparisons from HST data by both CMD techniques of three such clusters, Pal 3,4 and Eridanus, with the inner halo clusters M3 and M5, of similar metallicities. They found no evidence for an age spread among the three outer-halo clusters. At face value, their data suggest that those clusters could be younger by $\sim 1.5 \mathrm{Gyr}$ than their comparison clusters; a caveat in their result is that errors of 0.2 dex (which cannot be ruled out with present data) in $[\mathrm{Fe} / \mathrm{H}]$ or $[\alpha / \mathrm{H}]$ could reduce or eliminate the inferred small age difference between the two groups. Finally, two independent studies that compared CMDs for seven old, metal-poor GCs in the Large Magellanic Cloud with those of comparable clusters in the Galaxy failed to detect any age differences between them in excess of $\sim 1$ Gyr or so (Olsen et al. 1998, Johnson et al. 1999); constraints on the age range from such studies 
are also potentially limited by the quality of available spectroscopic abundance determinations for the LMC clusters.

In summary, there is evidence that a fraction of the intermediate-metallicity GCs in the Galactic halo may be younger than the bulk of the system. However, in recent years evidence from careful differential CMD comparisons suggests any age dispersion among the bulk of the Galactic GCs (or those in our distant halo) remains below the observational detection limit at a given metallicity, while the evidence for a mild age-metallicity relation remains a more debated question. It thus appears that the bulk of the Galactic GC system, and especially the most metal-poor ones, formed quickly during the earliest phases of Galactic history.

\section{When Did Formation Begin?}

We now turn to the more difficult question of absolute ages or, at what redshift did metal-poor star formation begin in the Galaxy? Recent work by Bergbusch \& VandenBerg (2001) interpreting the CMD of M92 with their new isochrones favors an age of $\sim 15-16$ Gyr. This would be reduced by $\sim 10 \%$ if He or heavyelement diffusion were incorporated into their models. Selection of the appropriate mixing-length for the comparison continues to require judgement. Three field halo subgiants they concurrently studied appeared also to have ages $\geq 15 \mathrm{Gyr}$.

Grundahl et al. (2000) found an M92 age of 15 Gyr using Strömgren color-color (c, c-y) diagrams, which have the powerful advantage of being distance-independent. However, their interpretation requires accurate knowledge of the $\mathrm{T}_{\text {eff }}$ scale, which they (and Bergbusch \& VandenBerg 2001) argue is well-established. They also compared M92 Strömgren color-color data with those of the field subdwarfs having $[\mathrm{Fe} / \mathrm{H}]<-2$ dex and concluded that the subdwarfs are the same age, 16 Gyr, as M92 before taking diffusion into account.

Carretta et al. (2000) studied nine clusters along with a large sample of subdwarfs with good parallax measurements, and concluded, after deciding that distances from the subdwarf fitting method are $\sim 1 \sigma$ too long, that the mean age of their sample was $12.9 \pm 2.9$ Gyrs (95\% confidence range). Further analysis in terms of cosmological models led them to conclude that the initial epoch of GC formation occurred around $\mathrm{z} \sim 3$.

Until now, the quality-both internal and external-of photometry has been a limiting factor in the quest for reliable absolute ages for GCs. Fig. 1 is a remarkable example of work Peter Stetson has been doing in the past year or so combining data from many observers and telescopes. This particular CMD is based upon 197(!) CCD images from four telescopes obtained during 15 nights and eight observing runs. The data all came from the original observers. Enabling such research constitutes a powerful argument for cataloguing and archiving data from ground-based telescopes, as most images taken since the introduction of CCDs are tragically languishing on deteriorating tapes in observers' offices worldwide. With photometric zero points now known to better than $1 \%$ on the new standard system (Stetson 2000), and with internal photometric scatter reduced to insignificance by combining many data, photometric quality will no longer limit the ability to determine accurate absolute and relative ages. 


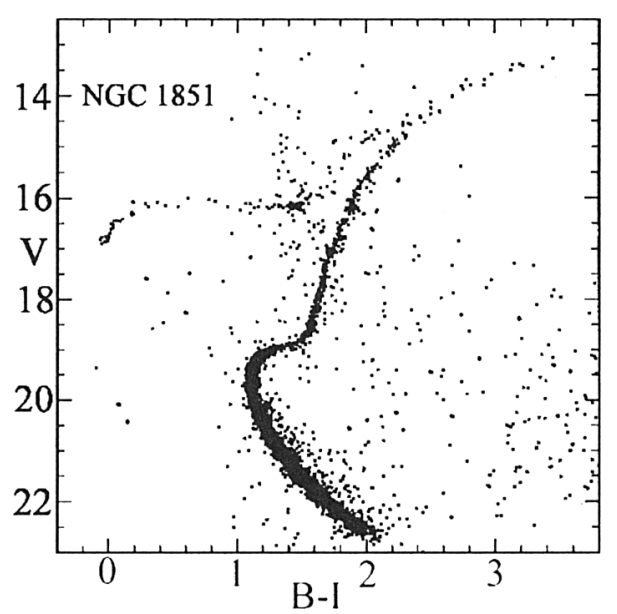

Figure 1. CMD by Stetson for NGC 1851 based upon 197 CCD images.

\section{What Limits Our Present Understanding?}

As photometric precision ceases to be a determining factor in the accuracy of age determinations, what factors remain important? Accurate knowledge of interstellar reddening for individual clusters and stars therein remains crucial, and nontrivial. Similarly, reducing uncertainties in distances from parallaxes remains a major challenge. Uncertainties in chemical composition determinations are a fundamental yet extremely challenging subject ripe for renewed efforts, as we now discuss. For instance, there are still controversies about the basic scale of GC abundances (Zinn \& West 1984; Carretta \& Gratton 1997; Rutledge, Hesser, \& Steton 1997). That highly experienced teams often find differences in overall abundances well beyond observational errors indicate that systematic errors still limit accuracy in high spectral resolution studies. Finally, abundances for the most distant objects often are inferred from broad-band photometry, or lowresolution spectra of $\alpha$-elements, like $\mathrm{Ca}$, in the most luminous giants, rather than from high-dispersion spectra of less evolved stars.

\subsection{Abundances from giants and dwarfs}

A question of considerable interest is whether inferences from more luminous cluster stars apply to the main-sequence and turnoff stars weighted so heavily in age determinations. Until the advent of $8-10-\mathrm{m}$ telescopes $\mathrm{HB}$ and red giant branch (RGB) stars have been the only ones for which abundances based upon higher spectral resolution were possible.

HB stars anchor distance moduli, underpin the LDZ interpretation of halo formation, and play many other important roles in our understanding of the galactic halo. An interesting example of modern abundance work is Behr, Cohen, \& McCarthy's (2000) study of the abundances of HB stars in M15, with comparisons to M13 (Behr et al. 1999) and NGC 6752 (Moehler et al. 1999). Among the slowly rotating hot stars two were found that exhibit the same ex- 
treme metal-deficiency that characterizes other M15 stars, but the other six stars studied show solar(!) $[\mathrm{Fe} / \mathrm{H}]$ values due to the radiative levitation of metals predicted by Michaud, Vauclair, \& Vauclair (1983). Many other interesting trends in detailed element abundances emerging from their study remind us how sensitively the HB reflects many parameters of stellar astrophysics.

The recognition of the phenomenon of abundance spreads among giant stars within an individual GC, but not among open cluster or field stars (e.g., Hesser, Hartwick, \& McClure 1976), sparked studies aimed at clarifying the percentage of the dispersion attributable to internal mixing during evolution up the giant branch, differences in initial abundances during the main-sequence phase, accretion, etc. Ivans et al. (1999) reported the range of individual chemical elements in the $[\mathrm{Fe} / \mathrm{H}]=-1.18 \pm 0.02$ dex cluster $\mathrm{M} 4$ determined from $\mathrm{R}=30,000$ or 60,000 spectral data with $\mathrm{S} / \mathrm{N}>100$ for 36 giants. Most of the scatter seen for elements heavier than $\mathrm{Al}$ is observational, but they found evidence for a wide range of $[\mathrm{O} / \mathrm{Fe}],[\mathrm{Na} / \mathrm{Fe}]$ and $[\mathrm{Al} / \mathrm{Fe}]$ within this cluster. Since both $[\mathrm{Fe} / \mathrm{H}]$ and $[\alpha / \mathrm{Fe}]$ play important roles in the age dating process, we need to understand how to characterize them for a given cluster when selecting appropriate isochrones.

Work with Keck and the VLT at higher resolution for fainter apparent magnitudes than heretofore possible using 4-m class telescopes (e.g., Cannon et al. 1998) allowed Gratton et al. (2001) to compare abundances derived from RGB and turnoff (TO) stars. Encouragingly, they found the same $<[\mathrm{Fe} / \mathrm{H}]\rangle$ values from stars in these different evolutionary states in the clusters NGC 6752 and 6397. They also infer an $\mathrm{O}-\mathrm{Na}$ anticorrelation among the main sequence stars, as previously seen among the giants in these clusters.

\section{2. $[\mathrm{O} / \mathrm{Fe}],[\mathrm{Si} / \mathrm{Fe}]$ in Cluster and Field Stars}

As the third most abundant element, oxygen plays an important opacity role in stellar atmospheres. Uncertainty in the $[\mathrm{O} / \mathrm{Fe}]$ ratio is a factor in determining the absolute ages of GCs via theoretical isochrone fits. VandenBerg (1992) determined that a 0.25 dex change in $[\mathrm{O} / \mathrm{Fe}]$ will change the resulting cluster age by 1 Gyr. While modern abundance analyses can obtain internal relative abundance uncertainties of less than $0.25 \mathrm{dex}$, the systematic offsets between the different measurement methods of determining oxygen abundances can be much larger.

Figure 2 plots $[\mathrm{O} / \mathrm{Fe}]$ abundances determined by three methods: the nearUV OH lines, the forbidden resonant [O I] lines in the optical, and the far red O I permitted triplet. Each method has strengths and drawbacks, as described in King (2000). As can be seen in Figure 2 the oxygen abundance ratio measures determined from the [O I] lines are significantly lower than the determinations derived from the $\mathrm{OH}$ and permitted lines. For the most metal-poor GCs, the difference is about 0.5 dex, which translates into a $\sim 2$ Gyr systematic uncertainty in the ages of these GCs. The GC oxygen abundances are determined from the forbidden line, which is relatively stronger in cool giants. The permitted and $\mathrm{OH}$ lines are stronger in hotter stars, and the sample of very metal-poor stars with both forbidden- and permitted-line oxygen abundance analyses is small.

Fulbright \& Kraft (1999), for example, found that the [O/Fe] ratio derived from [O I] line in two very metal-poor subgiants does not match the $[\mathrm{O} / \mathrm{Fe}]$ ratio derived from the $\mathrm{OH}$ or permitted lines in the same stars. Recent attempts to 


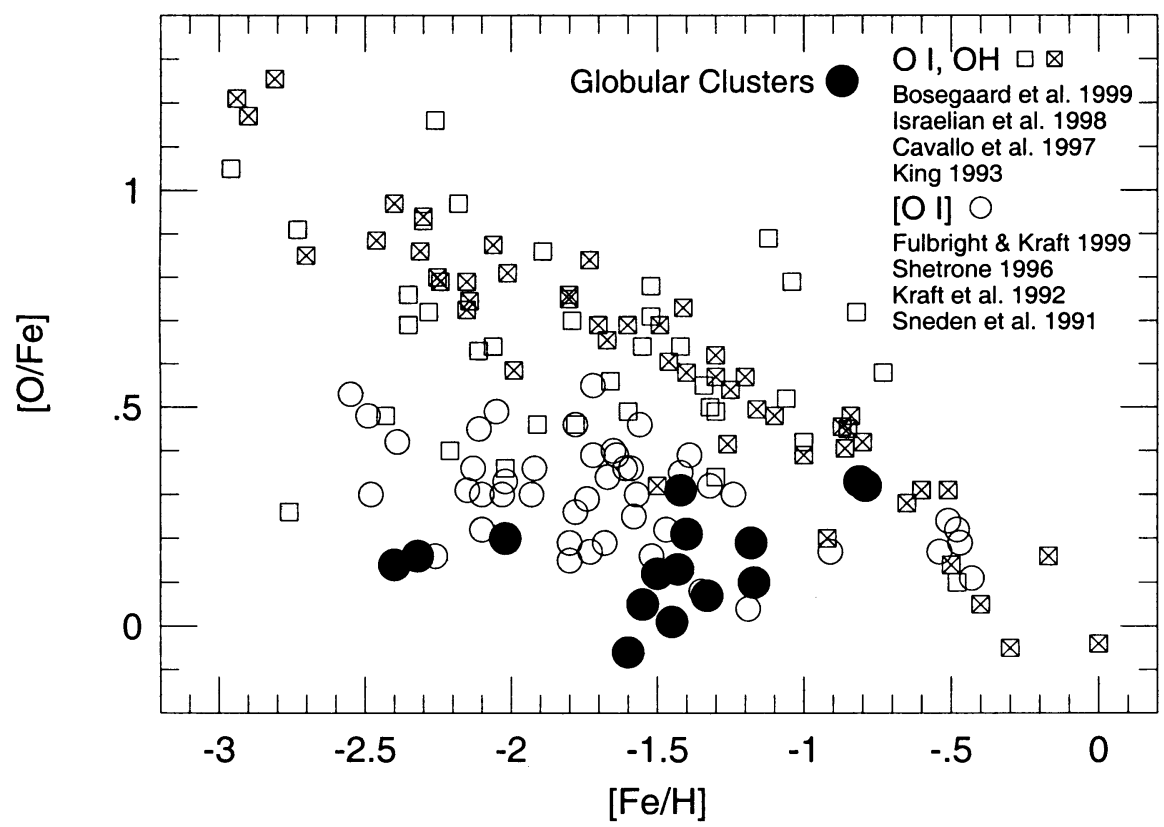

Figure 2. $\quad[\mathrm{O} / \mathrm{Fe}]$ ratios from several $\mathrm{GC}$ and field star studies.

resolve this discrepancy (King 2000, Israelian et al. 2001) have involved a careful examination of the atmospheric parameters used in the abundance analysis and the inclusion of non-LTE corrections. Asplund \& García Pérez (2001) suggests that full 3-D atmospheres may be necessary to model the oxygen lines correctly.

\section{3. $[\mathrm{Si} / \mathrm{Fe}]$ between GCs}

Silicon is the lightest $\alpha$-element that does not show large star-to-star abundance variations within individual metal-poor GCs. However there does seem to be a cluster-to-cluster scatter in [Si/Fe]. As first pointed out by Kraft (2000), studies of M4 (Ivans et al. 1999; 36 stars) and M5 (Sneden et al. 1992; 12 stars) found both to have similar values of $[\mathrm{Fe} / \mathrm{H}](-1.18 \mathrm{dex}$ and $-1.17 \mathrm{dex}$, respectively), but M4 is twice as Si-rich as M5 ([Si/Fe] ratios of +0.55 and +0.20 , respectively). Both studies were conducted by the same group using similar techniques, and the star-to-star scatter within the individual clusters is smaller than the clusterto-cluster difference.

This is evidence that there was a significant chemical inhomogeneity between the material that formed these clusters. Since the clusters are massive and on the metal-rich end of the halo metallicity distribution, it is unlikely that a random enrichment event would have had a sizable affect on the cluster's abundances. The lack of $[\mathrm{Si} / \mathrm{Fe}]$ variations within a cluster suggest that 


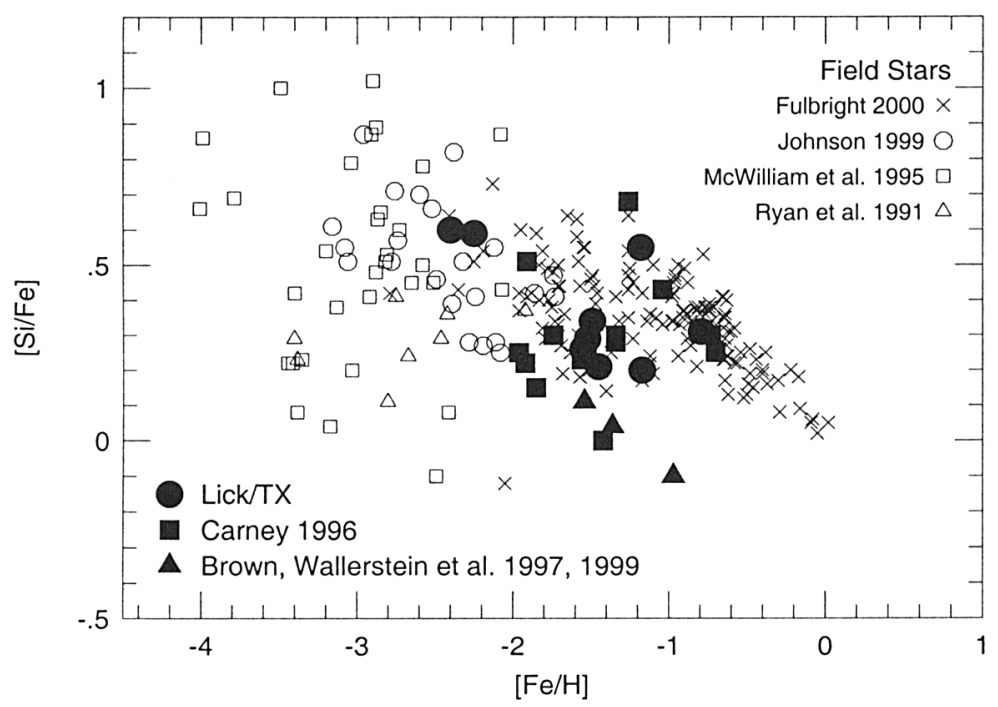

Figure 3. $\quad[\mathrm{Si} / \mathrm{Fe}]$ vs. $[\mathrm{Fe} / \mathrm{H}]$ ratios from $\mathrm{GC}, \mathrm{dSph}$ and field star studies.

the cluster-forming material was well-mixed prior to formation, so whatever led to the cluster-to-cluster variations in $[\mathrm{Si} / \mathrm{Fe}]$ most likely took place before the clusters formed.

In Figure 3 we plot the distribution of $[\mathrm{Si} / \mathrm{Fe}]$ vs $[\mathrm{Fe} / \mathrm{H}]$ for several globular clusters (mean values) and field stars. Aside from Ru 106 and Pal 12, there is general agreement in the range of $[\mathrm{Si} / \mathrm{Fe}]$ values seen in three samples. Two separate, parallel, tracks seem to be defined by M4 and the more metal-rich GCs and another by M5 and the metal-poor GCs. Whether this structure is truly there or is just a sampling effect is hard to determine from the data in hand. Nissen \& Schuster (1997) found that for field stars with $[\mathrm{Fe} / \mathrm{H}] \sim-1 \mathrm{dex}$, there are halo stars that show lower $[\alpha / \mathrm{Fe}]$ enhancements than disk and other halo stars at a similar metallicity. Fulbright (2001) shows evidence that for field halo stars with $-1>[\mathrm{Fe} / \mathrm{H}]>-2$, the stars having higher kinetic energy (e.g., on more extreme orbits) on average, show lower values of $[\mathrm{X} / \mathrm{Fe}]$ for several elements, including $[\mathrm{Si} / \mathrm{Fe}]$.

Dinescu et al. (1999) computed orbital parameters for $38 \mathrm{GCs}$ and found M4's apogalactic radius to be $\sim 6 \mathrm{kpc}$, while M5's is $\sim 40 \mathrm{kpc}$. Thus connection between kinematics and observed abundances for halo objects extends to M4 and M5, as well. The origin of such a relationship, however, is unclear. The age information we have suggests that all the metal-poor GCs formed at about the same time, so both of these processes must have occurred simultaneously, although one or both of the clusters could have formed in a separate fragment that was accreted later. 


\subsection{Th and U: Independent paths to ages}

An exciting new development in the study of GCs is the possibility that the long-lived radioisotopes of the r-process elements Th (14 Gyr half-life) and U (4.5 Gyr half-life) can be used to obtain ages for individual stars. Cowan et al. (1999) obtained a mean age of 15.6 4 4.6 Gyr for two field stars based on Th line strengths. Sneden et al. (2000) obtained a Th-based age for M15 of $14 \pm 3$ Gyr. Johnson \& Bolte (2001) also measured Th-based ages for several field stars plus the M92 giant VII-18. For VII-18, they obtained an age of $8.8 \pm 5.6$ Gyr.

A major source of the large uncertainties is the initial $\mathrm{Th}$ abundance for the stars. In these studies the models for the initial $r$-process production were scaled to the observed stable rare earth elemental abundances (eg. Eu). The mass differences between the rare earths and Th leaves room for sizable error.

Cayrel et al. (2001) successfully observed the $3895 \AA$ line of U II in the metal-poor field giant CS31082-001 and derived an age of $12.5 \pm 3$ Gyr for it. This star is special in that while the Fe and other light element abundances are about $1 / 800$ of solar, the r-process abundances are about 1/9 of solar. Having two radioactive species creates the advantageous situation where the observed $\mathrm{U} / \mathrm{Th}$ ratio can be compared to a theoretical initial $\mathrm{U} / \mathrm{Th}$ ratio without having to scale to other r-process elements. Due to their proximity in atomic mass, the theoretical initial $\mathrm{U} / \mathrm{Th}$ ratio should be more precise than scaling either to the lighter r-process elements (Goriely \& Clerbaux 1999).

Besides the dependence on quality theoretical r-process calculations, the radioactive age-dating technique could be improved by work in two areas. First, our knowledge of the oscillator strengths of Th II and U II need improvement. Cayrel et al. (2001) quote a 0.12 dex uncertainty in the U II $\log (\mathrm{gf})$ value they used. That systematic error can change the resulting age by $2.6 \mathrm{Gyr}$. It is hoped that the potential of this method to provide an independent age for the Galaxy will induce atomic physicists to improve the data.

Second is the need for near UV/blue-sensitive very high resolution spectrographs on 10-m or larger telescopes. While the present status of the field would not be possible without the excellent HIRES and UVES spectrographs, the near UV region of even these very metal-poor stars are still plagued with line blending at resolutions $\sim 50000-70000$. The situation would be vastly improved at resolutions $>150000$ with similar $\mathrm{S} / \mathrm{N}$ levels. The reduction of blending problems and the increased contrast against the continuum would help make measurements of the $\mathrm{Th}$ and $\mathrm{U}$ lines possible in GC stars, which have not been found to be as enhanced in the r-process elements as CS31081-001.

\section{Concluding Remarks}

True to Neruda's poetry, the pale, metal-deficient objects throughout the Galaxy's extended environs remain enigmatic, but they continue to yield important insights into how the Milky Way might have formed, and how that might in turn constrain understanding of galaxy formation in general. The salient points from our attempt to review the current status are:

1. The initial epoch of massive, metal-poor star cluster formation proceeded quickly $(<1.5-2.0$ Gyr); there are, however, younger clusters at intermediate- 
metallicities, which may have been accreted later. In particular, the most metaldeficient clusters (and field stars) show no discernible age dispersion throughout their 1-100 kpc extent in $\mathrm{R}_{\mathrm{gc}}$.

2. The most distant second-parameter GCs do not appear to be more than 1.5-2 Gyr younger than comparable metallicity, inner-halo clusters, but uncertain knowledge of their respective chemistry presents a caveat.

3. Absolute ages of the most metal-deficient clusters remain controversial with recent determinations falling in the range 12 to 16 Gyrs with uncertainties of $\sim 2-3$ Gyrs; recent consistency checks using abundances of radioactive elements (Th, U) are promising.

4. Existence of a putative age-metallicity relation also remains controversial; it presently seems likely to be $\lesssim 10 \%$.

5. Photometric precision will soon not be a limiting observational factor on the accuracy of cluster ages; rather, uncertainties in reddenings, distances and chemical composition will dominate. The time is ripe for major efforts on abundance determinations with 8-m and larger telescopes, but the most interesting problems may require telescopes with $20-30$-m aperture.

6 . There is growing evidence that dispersion of light elements within individual GCs reflect the epoch of star formation, rather than of mixing during stellar evolution; such differences combine with uncertain $[\mathrm{O} / \mathrm{Fe}]$ determinations to complicate accurate age determinations. It is encouraging that the average compositions determined from evolved and turnoff region stars seem to be the same within the limits of the data quality in the few clusters yet examined.

7. Cluster-to-cluster differences for ratios of light elements imply substantially different enrichment histories.

8. Field halo stars exhibit wide ranges of $[\mathrm{X} / \mathrm{Fe}]$; such differences provide detailed insight into early star formation.

9 . Over $\sim 5$ orders of magnitude in total mass (from GCs to dSphs to the LMC), the most metal-poor Local Group constituents appear to have formed at the same epoch, yet to also have been aware of their ultimate association with a particular parent.

Acknowledgments. We are grateful to Frank Grundahl, Jennifer Johnson, J.-W. Lee and Peter Stetson for sharing material that we used when preparing this paper, and to Eva Grebel and Doug Geisler for organizing an outstanding conference in a beautiful location.

\section{References}

Asplund, M. \& García Pérez, A.E. 2001, A\&A, 372, 601

Behr, B.B., Cohen, J.G. \& McCarthy, J.K. 2000, ApJ, 531, L37

Behr, B.B. et al. 1999, ApJ, 517, L135

Bergbusch, P.A. \& VandenBerg, D.A. 2001, ApJ, 556, 322

Bosegaard, A. et al. 1999, AJ, 117, 492

Brown, J.A., Wallerstein, G., \& Zucker, D. 1997, AJ, 114, 180

Brown, J.A., Wallerstein, G., \& Gonzalez, G. 1999, AJ, 118, 1245

Cannon, R.D. et al. 1998, MNRAS, 298, 601 
Carney, B.W. 1996, PASP, 108, 900

Carney, B.W. et al. 1996, AJ, 112, 668

Carney, B.W. 2001, in Saas-Fee Advanced Course 28, Star Clusters, ed. L. Labhardt \& B. Binggeli, Springer (Berlin), 1

Carretta, E. et al. 2000, ApJ, 533, 215

Caretta, E. \& Gratton, R.G. 1997, A\&AS, 121, 95

Cavallo, R.M., Pilachowski, C.A., \& Rebolo, R. 1997, PASP, 109, 226

Cayrel, R. et al. 2001, Nature, 409, 691

Cowan, J.J. et al. 1999, ApJ, 521, 194

Dinescu, D.I. et al. 1999, AJ, 117, 1792

Fulbright, J.P. 2000, AJ, 120, 1841

Fulbright, J.P. 2001, AJ, submitted

Fulbright, J.P. \& Kraft, R.P. 1999, AJ, 118, 527

Gonzalez, G. \& Wallerstein, G. 1998, AJ, 116, 765

Goriely, S. \& Clerbaux, B. 1999, A\&A, 346, 798

Gratton, R. et al. 2001, A\&A, 369, 87

Grundahl, F. 2001, private communication

Grundahl, F. et al. 2000, AJ, 120, 1884

Harris, W.E. 2001, in Saas-Fee Advanced Course 28, Star Clusters, ed. L. Labhardt \& B. Binggeli, Springer (Berlin), 223

Harris, W.E. et al. 1997, AJ, 114, 1030

Hartwick, F.D.A. 2000, AJ, 119, 2248

Hesser, J.E., Hartwick, F.D.A. \& McClure, R.D. 1976, ApJ, 207, L113

Israelian, G., García López, R. J. \& Rebolo, R. 1998, ApJ, 507, 805

Israelian, G. et al. 2001, ApJ, 551, 833

Ivans, I.I. et al. 1999, AJ, 118, 1273

Johnson, J.A. 1999, PhD Thesis, University of California, Santa Cruz

Johnson, J.A. et al. 1999, ApJ, 527, 199

Johnson, J.A. \& Bolte 2001, ApJ, 554, 888

King, J.R. 1993, AJ, 106, 1206

King, J.R. 2000, AJ, 122, 1056

Kraft, R.P. 2000, in IAU Joint Discussion 5, Mixing and Diffusion in Stars: Theoretical Predictions and Observational Constraints, in press.

Kraft, R.P. et al. 1992, AJ, 104, 645

Lee, J.-W. et al. 2001, private communication

Lee, Y.-W., Demarque, P., \& Zinn, R. 1994, ApJ, 423, 248

Martí nez Roger, C., Pérez Fournón, I., \& Sánchez, F. (Eds.), Globular Clusters, Cambridge University Press, 1999

McWilliam, A. et al. 1995, AJ, 109, 2757

Michaud, G., Vauclair, G. \& Vauclair, S. 1983, ApJ, 267, 256

Moehler, S. et al. 1999, A\&A, 346, L1 
Nissen, P.E. \& Schuster, W.J. 1997, A\&A, 326, 751

Olsen, K.A.G. et al. 1998, MNRAS, 300, 665

Prochaska, J.X., \& Wolfe, A.M. 2000, ApJ, 553, L5

Rosenberg, A. et al. 1999, AJ, 118, 2306

Ryan, S. G., Norris, J. E. \& Bessell, M. S. 1991, AJ, 102, 303

Rutledge, G.A., Hesser, J.E. \& Stetson, P.B. 1997, PASP, 109, 907

Shetrone, M.D. 1996, AJ, 112, 1517

Shetrone, M.D., Côté, P., \& Sargent, W.L.W. 2001, ApJ, 548, 592

Sneden C. et al. 1991, AJ, 102, 2001

Sneden C. et al. 1992, AJ, 104, 2121

Sneden C. et al. 2000, ApJ, 536, 85

Stetson, P.B. 2000, PASP, 112, 925

Stetson, P.B. et al. 1999, AJ, 117, 247

Stetson, P.B., VandenBerg, D.A., \& Bolte, M. 1996, PASP, 108, 560

VandenBerg, D.A. 1992, ApJ, 391, 685

VandenBerg, D.A. 2000, ApJS, 129, 315

van den Bergh, S. 1996, PASP, 108, 986

van den Bergh, S. 2000a, ApJ, 530, 777

van den Bergh, S. 2000b, PASP, 112, 932

Zinn, R., \& West, J.J. 1984, ApJS, 55, 45

\section{Discussion}

$P$. Demarque: Is it not true that VandenBerg's ages do not include the effects of He diffusion? We know that diffusion takes place in the sun, from helioseismology, and it is believed to be more efficient in halo stars.

$J$. Hesser: VandenBerg's current analysis does not include diffusion yet, and he suggests that inclusion of diffusion will lower his age estimates about $10-15 \%$.

D'Antona: Recently, Gratton et al. have found that the iron and oxygen abundances in NGC 6397 turnoff stars are equal to subgiant branch abundances, although models including diffusion predict differences, also by a factor of two, for this metal-poor cluster. Of course, this does not give information on helium diffusion, which affects the age determinations, but is an indicator that the models are not adequate for the diffusion of metals.

G. Wallerstein: Considering diffusion, could Fe diffuse downward so that our measured Fe abundances in the atmosphere not represent the Fe abundance in the deep interior?

F. D'Antona: $\mathrm{Fe}$ diffusion is not important so the measured $\mathrm{Fe}$ abundances should be correct. 